\title{
The creeping motion of liquid drops through a circular tube of comparable diameter
}

\author{
By B. P. HO AND L. G. LEAL \\ Chemical Engineering, California Institute of Technology, Pasadena
}

(Received 13 January 1975)

The creeping motion through a circular tube of neutrally buoyant Newtonian drops which have an undeformed radius comparable to that of the tube was studied experimentally. Both a Newtonian and a viscoelastic suspending fluid were used in order to determine the influence of viscoelasticity. The extra pressure drop owing to the presence of the suspended drops, the shape and velocity of the drops, and the streamlines of the flow are reported for various viscosity ratios, total flow rates and drop sizes.

\section{Introduction}

The experimental study reported in this paper is concerned with the creeping motion of neutrally buoyant drops of a Newtonian fluid through a straight circular tube when the diameters of the undeformed drops and the tube are of similar magnitude. Both Newtonian and viscoelastic suspending fluids have been considered in the present work. Much of the earliest interest in these problems arose because of the suggested analogy in the Newtonian case between the drop motion and the motion of erythrocytes in the capillaries. Although subsequent work on erythrocyte motion has now largely eliminated this motivation for investigation, the motion of a drop or train of drops through a tube of constant or variable cross-sectional area remains of considerable technological importance in its own right. One specific example where the case of a viscoelastic suspending fluid is of special significance is the motion of oil droplets in a porous matrix during tertiary (oil) recovery processes which use polymeric 'pusher' fluids, e.g. micelle or polymer flooding (see Savins 1969).

In the present experiments, we have determined the change in the pressure drop $\Delta P^{+}$which is required, in the presence of the suspended drops, to produce a given total volumetric flow rate $Q$, the drop shape, the velocity $U$ of the drops relative to the average velocity $\bar{V}$ of the two-phase system as a whole, and finally, the streamline patterns with respect to a frame of reference in which the drops are stationary. The independent variables which were varied for both the Newtonian and viscoelastic suspending fluids are the total volumetric flow rate $Q$, the volume $v$ of the suspended drops and the ratio $\sigma$ of the shear viscosity $\mu_{i}$ of the suspended fluid to that of the suspending fluid, $\mu_{0}$. For the case of a Newtonian system in the absence of fluid inertia effects, the appropriate characteristic pressure is simply $\mu_{0} \bar{V} / R_{0}$, where $R_{0}$ is the radius of the tube. The extra pressure drop $\Delta P^{+}$, 
non-dimensionalized by $\mu_{0} \bar{V} / R_{0}$, is then a function of (i) the relative size of the drop as measured by the ratio $\lambda$ of the undeformed drop radius to the tube radius, (ii) the relative viscosity $\sigma=\mu_{i} / \mu_{0}$ and (iii) a deformation parameter $\Gamma=\mu_{0} \bar{V} / \gamma$, where $\gamma$ is the interfacial tension between the suspending fluid and the drop. When the suspending fluid is viscoelastic, additional parameters which measure the degree of elasticity are required.

So far as we are aware, no previous investigation has considered the case of a viscoelastic suspending fluid. However there have been several experimental and theoretical studies of the creeping motion of both drops and solid particles through a circular tube when the suspending fluid is Newtonian. On the theoretical side, the investigations which are most relevant to the present work are those of Hetsroni, Haber \& Wacholder (1970), Brenner (1971) and Hyman \& Skalak (1972a,b). Hetsroni et al. (1970) used the method of reflexions to solve for the flow fields in and around a single undeformed drop suspended in a Poiseuille flow. Their result for the velocity of a neutrally buoyant concentrically located spherical drop, which is valid for small values of $\lambda$, is

$$
U / \bar{V}=2-\frac{4 \sigma}{3 \sigma+2} \lambda^{2}+O\left(\lambda^{3}\right)
$$

Since the drop was assumed spherical, the boundary condition on the normal component of the stress is not satisfied. However, upon substitution of the velocity fields for an undeformed drop, this boundary condition gives a first approximation to the deformed shape of the drop. The result obtained by Hetsroni et al. (1970) for the case of a neutrally buoyant concentrically located drop is

$$
r=r_{0}\left[1+\frac{1}{2} L_{3}^{0}\left(5 \cos ^{3} \theta-3 \cos \theta\right)\right]+O\left(\lambda^{3}\right),
$$

where

$$
L_{3}^{0}=\frac{10+11 \sigma}{10(1+\sigma)} \Gamma \lambda^{2}
$$

which is valid provided that $\sigma=O(1)$ and $\Gamma \rightarrow 0$. Here $r_{0}$ is the radius of the undeformed drop, $r$ is the radial variable measured relative to the centre of the drop and $\theta$ is the polar angle measured counterclockwise from the axis of the undisturbed velocity vector. It should be noted that the deviation from a spherical shape is predicted to increase linearly with $\Gamma$, and also to increase slightly with an increase in the viscosity ratio $\sigma$. Hetsroni et al. (1970) did not obtain results for the extra pressure drop caused by the presence of the drop. However, shortly thereafter, Brenner (1971) obtained this quantity using the reciprocal theorem for low Reynolds number flow. The result for a neutrally buoyant concentrically located spherical drop is

$$
\frac{\Delta P^{+} R_{0}}{\mu_{0} \bar{V}}=\frac{16\left[(9 \sigma+2)^{2}-40\right]}{27(\sigma+1)(3 \sigma+2)} \lambda^{5}+O\left(\lambda^{10}\right) .
$$

It is significant, as Brenner has pointed out, that $\Delta P^{+}$may be either positive or negative (i.e. it is predicted that the overall pressure drop can be either increased or decreased by the presence of the drop) depending on the magnitude of $\sigma$. In addition, although (3) is strictly valid only for $\lambda \ll 1$, the very small relative error 
would appear to allow quite reasonable results for values of $\lambda$ as large as $0 \cdot 3-0 \cdot 4$.

For higher values of $\lambda$, Hyman \& Skalak $(1972 a, b)$ studied the case of an equally spaced train of neutrally buoyant concentrically located drops including both deformed and undeformed shapes. Although exact solutions of the equations of motion were derived, these were in the form of an infinite series of algebraic equations. Hence, in order to obtain quantitative (numerical) results, it was necessary to truncate the series and restrict the parameters $\sigma$ and $\Gamma$ and particle spacing to specific values, while keeping $\lambda \leqslant 0 \cdot 8$. For $\lambda>0 \cdot 8$, the number of algebraic equations required for convergence became excessive, even with the shape specified. Moreover the actual deformation from a spherical shape became so large for $\lambda>0.8$ that many trials would have been required to obtain the correct equilibrium shape of the drop. Comparison of the results of Hyman \& Skalak (1972a) for the velocity of a single undeformed drop with (1) indicates agreement to three significant figures for $\lambda<0 \cdot 4$ but increasing deviation for larger values of $\lambda$, presumably due to the neglect of higher-order terms in $\lambda$ in (1). At $\lambda=0 \cdot 7$, the velocities predicted by (1) exceed the values calculated by Hyman \& Skalak (1972a) by $3 \%$. At $\lambda=0 \cdot 8$, the difference is $6 \%$. Likewise comparison of $\Delta P^{+}$from Hyman \& Skalak (1972a) with that predicted by Brenner's theory, (3), shows that the latter increasingly underpredicts the former with increasing $\lambda$. At $\lambda=0.5$ the difference is $5 \%$.

On the experimental side, Sutera \& Hochmuth (1968) and Hochmuth \& Sutera (1970) have investigated the case of solid hemispheres and caps in a Newtonian suspending fluid as a model for the motion of erythrocytes through capillaries. In addition, Prothero \& Burton $(1961,1962)$ have reported a more or less qualitative investigation of a train of gas bubbles in a Newtonian fluid as a model for blood flow through capillaries. However the only investigations of direct relevance to the present paper are those of Goldsmith \& Mason (1963) and Bretherton (1961), who studied very large $(\lambda \gg 1)$ suspended drops in slow motion $(\Gamma \ll 1)$ where the drop and tube wall are separated by only a thin layer of suspending fluid. The variables measured in these studies were mainly the velocity of the fluid drop relative to the average fluid velocity, and the thickness of the thin layer of fluid separating bubble and wall. The chief result of qualitative interest here is the occurrence of an apparent asymptotic behaviour for large $\lambda$ in which the velocity of the drop and thickness of the thin layer of fluid become independent of $\lambda$. No measurements of $\Delta P^{+}$were made.

The present investigation covers the range $\lambda \sim 1$ for intermediate values of $\Gamma$, and $\sigma$ varying from approximately $0 \cdot 2$ to 2 . Thus, for the Newtonian case, our results lie between the available theory $(\lambda \lesssim 0 \cdot 8)$ and experiments $(\lambda \gg 1)$. Also, we provide an initial study of the additional effects associated with a viscoelastic suspending fluid. The range of $\lambda$ near unity is of considerable interest since it is here that the maximum variations in drop shape and wall effect on $\Delta P^{+}$may be expected. 


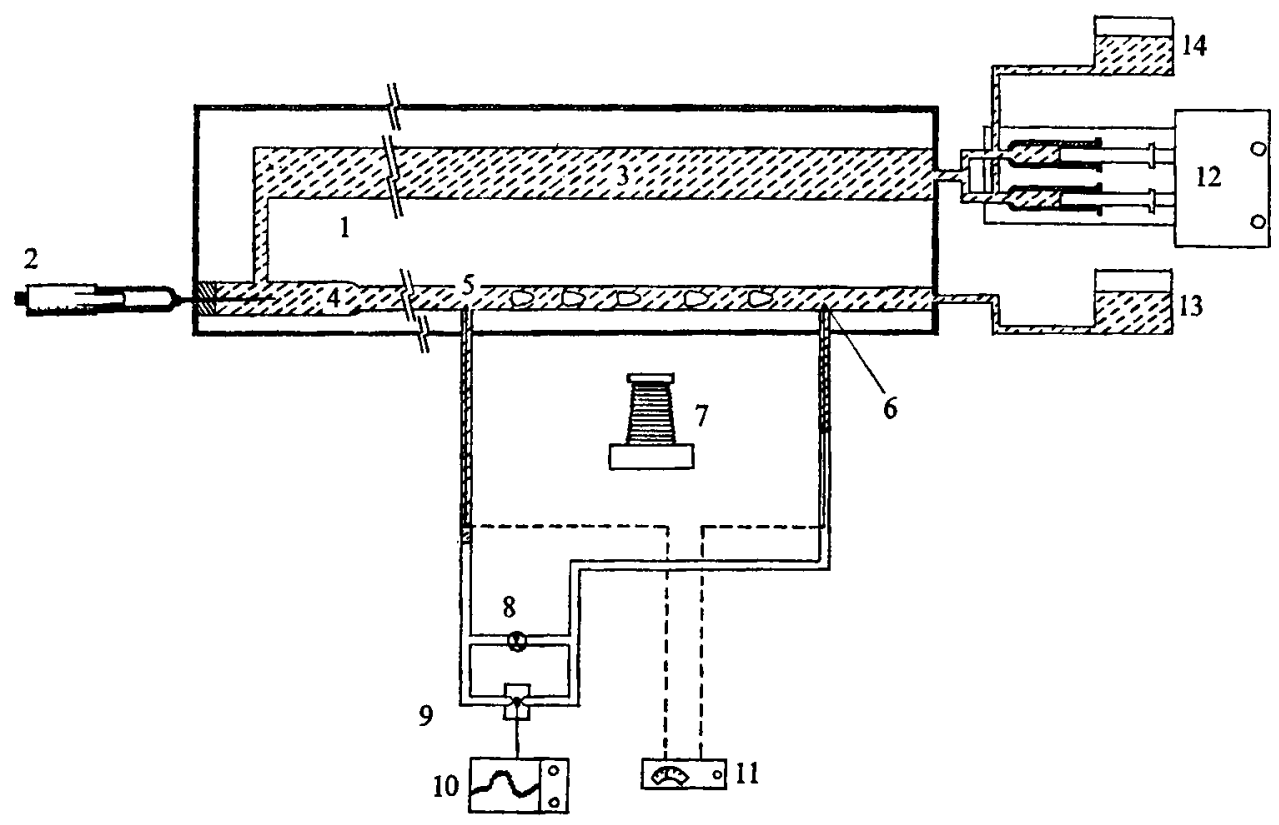

Figure 1. Schematic diagram of experimental set-up (not to scale). (1) Constant-temperature bath. (2) Micrometer syringe. (3) Bulk-fluid storage. (4) Test section. (5) Pressure hole. (6) Thermocouple probe. (7) Camera and moving mechanism. (8) By-pass valve. (9) Pressure transducer. (10) Transducer indicator and recorder. (11) Thermocouple reading. (12) Withdrawal and infusion pump. (13) Waste storage. (14) Storage reservoir.

\section{Apparatus and experimental techniques}

\subsection{Experimental set-up}

The experimental set-up is shown schematically in figure 1. The test section, where all the data were obtained, consisted of a horizontal precision-bore glass tube $120 \mathrm{~cm}$ long of internal diameter $\left(2 R_{0}\right) 1 \mathrm{~cm}$. Two pressure taps were located $50 \mathrm{~cm}$ apart, the tap further upstream being approximately $50 \mathrm{~cm}$ from the entrance to the $1 \mathrm{~cm}$ tube. These pressure taps were connected to a manometer which was conventional except for a valve at the bottom which could be closed to allow the pressure difference between the bottoms of the two legs to be measured and recorded using a differential pressure transducer. The method of obtaining $\Delta P^{+}$with this set-up will be described later in this section. The $50 \mathrm{~cm}$ section of tube upstream of the first pressure tap and the $20 \mathrm{~cm}$ section downstream of the second were intended to minimize end effects. At its upstream end, the test section was connected to a larger $(1.4 \mathrm{~cm}$ diameter $)$ glass tube into which the drops were manually injected using a precision micrometer syringe (accurate to within $\pm 0.001 \mathrm{ml}$ ) connected to a 17 gauge hypodermic needle. The larger diameter tube was adopted in order to facilitate the injection of the larger drops $(\lambda>1)$ after it was found that the injection method inevitably brought the drops into contact with the wall of the smaller $(1 \mathrm{~cm})$ tube, where they became permanently affixed owing to the wetting properties of the drop liquid. The 
1 and $1.4 \mathrm{~cm}$ glass tube were both enclosed in a long Plexiglas constanttemperature bath.

The suspending fluid was pumped in at a constant flow rate using a variablespeed Harvard Apparatus reciprocal-action infusion-withdrawal syringe pump. While one syringe was supplying the suspending fluid into the test section by injection, the other was being filled from a reservoir by withdrawal, thusallowing a continuous flow to be maintained. A large storage section was connected to the exit of these syringes and placed inside the constant-temperature bath to ensure that the suspending fluid was at the bath temperature $\left(25 \cdot 0 \pm 0.5{ }^{\circ} \mathrm{C}\right)$ before entering the test section. Two thermocouples, one at each pressure tap, were used to monitor the temperature of the suspending fluid in the test section. The drops were injected one by one with the pump completely turned off. After a given volume of the drop fluid had been injected, bulk fluid was pumped in slowly so that the drop detached itself from the hypodermic needle. The drops were found to migrate to the concentric position as long as they were neutrally buoyant, so that no special effort was made to inject them onto the centre-line of the tube. The tendency of small neutrally buoyant drops to move to the tube axis had been previously reported by Karnis \& Mason (1967). When trains of several drops were considered, as was most often the case, the drops were injected such that they were as evenly spaced as possible. However it was observed here, and also predicted by Hyman \& Skalak $(1972 a, b)$, that interactions between drops were negligible provided that the distance between their centres was only slightly larger than the tube diameter. As all our data were taken with the spacing at least this large, the precision of the equal spacing was not critical to the results.

\subsection{Materials}

For the Newtonian case, the suspending fluid was $95 \cdot 75 \%$ by weight glycerine in water. The percentage of glycerine was monitored during the experiments by using a hydrometer to measure the solution density at $25 \cdot 0^{\circ} \mathrm{C}(1.251 \mathrm{~g} / \mathrm{c} . \mathrm{c}$. $)$. The viscosity was measured using a Canon-Fenske capillary viscometer and was consistently found to be within $2 \%$ of the published value of $417 \mathrm{cP}$ at $25^{\circ} \mathrm{C}$. It is believed that the small ( $2 \%$ ) discrepancies can be attributed to slight variations in the bath temperature for the viscometer, which could not be controlled more accurately than to within $\pm 0 \cdot 1^{\circ} \mathrm{C}$. It is well known that the viscosity of glycerine exhibits a rather strong dependence on temperature. For example, a $0.5^{\circ} \mathrm{C}$ change from $25^{\circ} \mathrm{C}$ produces a $5 \%$ change in the viscosity. Indeed, when an attempt was made to calibrate the experimental set-up of figure 1 by using the measured flow rate and pressure drop to determine the viscosity, it was found that only approximately $5 \%$ agreement with values in the literature could be obtained. This discrepancy presumably reflects the accuracy of temperature control, which was to within $\pm 0.5^{\circ} \mathrm{C}$ in the test section.

The viscoelastic suspending fluid was a $0.5 \%$ by weight solution of Dow Separan AP30 (an ionic polyacrylamide) in water. The viscosity and primary normal-stress difference for this material have been reported as a function of the shear rate by Leal, Skoog \& Acrivos (1971). Other properties, such as relaxation 
and retardation times or the elongational viscosity, which are required to characterize the material behaviour in a time-dependent non-viscometric flow are also available in the literature (Huppler, Ashare \& Holmes 1967; Huppler, MacDonald, Ashare, Spriggs, Bird \& Holmes 1967). However, since the present experiments encompass only a single polymer concentration, we shall not consider the various viscoelastic parameters further, with the exception of the shear viscosity $\mu_{0}$, which can be compared directly with the Newtonian case. Since the viscosity of $0.5 \%$ Separan is strongly shear-rate dependent and the flow through a tube (with or without drops) has a non-uniform shear rate, there is some arbitrariness in the precise value of $\mu_{0}$ to assign for a particular flow rate. However, in view of the fact that one of the major variables of interest is the additional pressure drop relative to that of the pure suspending fluid, we have used the apparent viscosity of the viscoelastic fluid at the wall shear rate of the pure suspending fluid which is appropriate for each volume flow rate. Thus, in order to obtain the apparent viscosity as a function of the volume flow rate, the pressure drop $L$ across the $50 \mathrm{~cm}$ test section was measured for various flow rates $Q$. For a unidirectional flow of any fluid, a simple force balance shows that the wall shear stress $\tau_{w}$ is related to the pressure drop by

$$
\tau_{w}=\frac{1}{2} R_{0}(\Delta P / L) .
$$

Furthermore it can be shown (see Coleman, Markovitz \& Noll 1966, p. 46) that the wall shear rate is given for any fluid by

$$
\beta_{w}=\frac{3 n+1}{4 n}\left(\frac{4 Q}{\pi R_{0}^{3}}\right)
$$

where $n$ is the slope of $\tau_{w}$ vs. $4 Q / \pi R_{0}^{3}$ on a $\log -\log$ plot. Although $n$ may generally vary with the flow rate, we have found that $n=0.450$ provides a good approximation to the viscosity of $0.5 \%$ Separan in the range of shear rates characterizing the present experiments. Thus, for our present purposes, the relationship between the apparent viscosity, $\mu_{0}\left(\equiv \tau_{w} / \beta_{w}\right)$ and $\beta_{w}$ can be represented by a power-law model. Values of $\mu_{0}$ for various flow rates are given in table 1 , together with other pertinent information on the conditions of our experiments. Values from the literature obtained in a simple shear viscometer are found to be slightly lower than those obtained by the present method, but the slope of $\mu_{0} v s . \beta_{w}$ is the same. Although no normal-stress measurements were made in the present study, we have also listed values of the first normal-stress difference at each $\beta_{w}$, from the work of Leal et al. (1971) in table 1.

The suspended drops consisted of a well-mixed solution of silicone oil (Dow Corning 200 fluid, a dimethyl silioxane polymer) and carbon tetrachloride which behaves as a Newtonian fluid in the range of shear rates of the present experiments. The two liquids were mixed in such a proportion that the density matched that of the suspending fluid to within $0.001 \mathrm{~g} /$ c.c. For the viscoelastic system, a mixture of about 18 parts of silicone oil to one part of carbon tetrachloride yielded a density (1.000 g/c.c.) equal to that of the Separan AP 30/water solution. For the Newtonian system, a mixture of about 12 parts of silicone oil to 10 parts of carbon tetrachloride gave a density $(1.251 \mathrm{~g} / \mathrm{c.c}$.) equal to that of the 


\begin{tabular}{|c|c|c|c|c|c|}
\hline & \multirow{2}{*}{$\begin{array}{l}\text { Quantities } \\
\text { independent } \\
\text { of flow rate }\end{array}$} & \multicolumn{4}{|c|}{$\begin{array}{l}\text { Quantities dependent } \\
\text { on flow rate }\end{array}$} \\
\hline & & (a) & (b) & (c) & $(d)$ \\
\hline Flow rate, $V(\mathrm{~cm} / \mathrm{s})$ & - & 0.390 & 0.525 & 0.756 & 0.920 \\
\hline \multicolumn{6}{|l|}{ Newtonian systems } \\
\hline Wall shear rate, $\beta_{w}\left(\mathrm{~s}^{-1}\right)$ & - & $3 \cdot 13$ & $4 \cdot 20$ & 6.05 & $7 \cdot 36$ \\
\hline Suspending-fluid viscosity, $\mu_{0}(\mathrm{P})$ & $4 \cdot 25$ & - & - & 一 & - \\
\hline Bulk Reynolds number, $\left(\rho V R_{0}\right) / \mu_{0}$ & - & 0.05 & 0.06 & 0.09 & $0 \cdot 1$ \\
\hline Deformation parameter, $\Gamma^{-1}$ & - & $13 \cdot 3$ & $9 \cdot 9$ & $6 \cdot 8$ & $5 \cdot 6$ \\
\hline \multicolumn{6}{|l|}{ Viscosity ratio, $\sigma$} \\
\hline System $1\left(\mu_{i}=8.65 \mathrm{P}\right)$ & $2 \cdot 04$ & - & - & - & - \\
\hline System $2\left(\mu_{i}=3.97 \mathrm{P}\right)$ & 0.93 & - & - & - & - \\
\hline System $3\left(\mu_{i}=2.45 \mathrm{P}\right)$ & 0.58 & - & - & - & - \\
\hline System $4\left(\mu_{i}=0.80 \mathrm{P}\right)$ & $0 \cdot 19$ & - & - & - & - \\
\hline \multicolumn{6}{|l|}{ Viscoelastic systems } \\
\hline Wall shear rate, $\beta_{w}\left(\mathrm{~s}^{-1}\right)$ & - & $4 \cdot 10$ & $5 \cdot 50$ & $7 \cdot 93$ & $9 \cdot 65$ \\
\hline Suspending-fluid viscosity, $\mu_{0}$ (P) & - & $6 \cdot 54$ & $5 \cdot 46$ & $4 \cdot 47$ & $4 \cdot 08$ \\
\hline $\begin{array}{l}\text { Suspending-fluid first normal- } \\
\left.\text { stress difference, } N_{1} \text { (dyne } / \mathrm{cm}^{2}\right)\end{array}$ & 一 & $40 \cdot 0$ & $50 \cdot 0$ & $70 \cdot 0$ & $80 \cdot 0$ \\
\hline Bulk Reynolds number, $\left(\rho V R_{0}\right) / \mu_{0}$ & - & 0.03 & 0.05 & 0.09 & $0 \cdot 1$ \\
\hline Deformation parameter, $\Gamma^{-1}$ & - & $14 \cdot 9$ & $13 \cdot 3$ & $11 \cdot 2$ & $10 \cdot 1$ \\
\hline \multicolumn{6}{|l|}{ Viscosity ratio, $\sigma$} \\
\hline System $5\left(\mu_{i}=20.1 \mathrm{P}\right)$ & - & $3 \cdot 1$ & $3 \cdot 7$ & $4 \cdot 5$ & $4 \cdot 9$ \\
\hline System $6\left(\mu_{i}=6.9 \mathrm{P}\right)$ & - & $1 \cdot 1$ & $1 \cdot 3$ & 1.5 & $1 \cdot 7$ \\
\hline System $7\left(\mu_{i}=2 \cdot 4 \mathrm{P}\right)$ & - & 0.37 & $0 \cdot 44$ & 0.54 & 0.59 \\
\hline System $8\left(\mu_{i}=0.69 \mathrm{P}\right)$ & - & $0 \cdot 11$ & $0 \cdot 13$ & $0 \cdot 15$ & $0 \cdot 17$ \\
\hline
\end{tabular}

TABLE 1. Conditions of experiments

hydrolysed glycerine. Four grades of silicone oil having different viscosities but equal density were used for the Newtonian system and will be labelled systems 1-4. Another four grades of silicone oil were used for the viscoelastic system and will be labelled systems 5-8. The viscosity of the mixture of silicone oil and carbon tetrachloride was measured by a Canon-Fenske capillary viscometer at $25 \cdot 0{ }^{\circ} \mathrm{C}$. An appropriate dimensionless parameter is the viscosity ratio $\sigma=\mu_{i} / \mu_{0}$. The viscosity of glycerine is constant while that of Separan in water is flow-rate dependent. Consequently $\sigma$ is also flow-rate dependent for the viscoelastic system. The viscosity $\mu_{i}$ of the mixture of silicone oil and carbon tetrachloride and the viscosity ratios for the eight systems are given in table $\mathbf{1}$ for the various flow rates used in the experiments.

\subsection{Conditions of the experiments}

Both the experiments for the Newtonian and for the viscoelastic case were thus carried out for four different combinations of the suspending and drop fluids, corresponding to systems 1-8. In addition, for each system of fluids, we used four different volume flow rates, and at each flow rate, six different volumes $v$ for the suspended drops. The various flow rates are labelled $(a)-(d)$, with $(a)$ 


\begin{tabular}{lllllll}
$v(\mathrm{ml})$ & 0.2 & 0.3 & 0.4 & 0.5 & 0.6 & 0.7 \\
$\lambda$ & 0.726 & 0.831 & 0.914 & 0.985 & 1.046 & 1.102 \\
& \multicolumn{7}{c}{ TABLE 2 } \\
& \multicolumn{3}{c}{ TAB }
\end{tabular}

corresponding to the lowest. They were chosen such that the bulk Reynolds number $R e=\rho \bar{V} R_{0} / \mu_{0}$ is small in every case. The actual values of $R e$ (the maximum being $0 \cdot 1$ ) are listed in table 1 . The six different drop volumes $v$ and corresponding $\lambda$ are given in table 2 .

At low Reynolds number, the parameter which characterizes the degree of drop deformation is the ratio $\Gamma=\mu_{0} \bar{V} / \gamma$, which is a measure of the relative importance of the viscous stresses and the interfacial tension $\gamma$. The interfacial tension, measured using a DuNoüy platinum ring tensiometer, was found to be 22 dyne/cm for an interface between the glycerine and the corresponding mixture of silicone oil and carbon tetrachloride, while for an interface between the Separan solution and the appropriate mixture of silicone oil and carbon tetrachloride it was found to be $38 \mathrm{dyne} / \mathrm{cm}$. Although $\gamma$ was thus fixed by the choice of materials, the parameter $\Gamma$ was varied through the choice of various flow rates $\bar{V}$ (and various values of $\mu_{0}$ for the viscoelastic case). In fact, the primary reason for varying $Q$ in the experiments was to study the effects of the flow rate on drop deformation.

\subsection{Methods of measurement}

Pressure measurements were made using a combination of a standard U-tube manometer, which was attached to the pressure taps in the test section, and a differential variable-reluctance pressure transducer and indicator system manufactured by Validyne Engineering Corporation. In order to minimize transients and other anomalies associated with the motion of the drops directly above the pressure taps, they were located $50 \mathrm{~cm}$ apart. This rather large separation was designed not only to allow the drop or train of drops to be completely contained between the two taps, but also to ensure adequate time in this 'enclosed' configuration for the measured pressure difference to attain a steady value. The major difficulty associated with the wide separation of the pressure taps was that the overall pressure drop was quite large, considerably exceeding the extra pressure drop $\Delta P^{+}$, which was the main pressure variable of interest. In order to achieve reasonable accuracy for the small change $\Delta P^{+}$, we used the combination of a manometer and transducer mentioned above. The manometer was allowed to come to equilibrium with the suspending fluid alone moving through the test section at the desired flow rate $Q$, and the valve at the bottom of the manometer was then closed, which effectively separated the two legs. In this configuration, the pressure transducer, which had a full-scale range of \pm 1 in. of water at $25^{\circ} \mathrm{C}$ could be used to measure accurately the differences between the pressures at the two taps which arose because of the presence of the drops in the test section (i.e. measure $\Delta P^{+}$directly). Carbon tetrachloride was chosen as the 
manometer fluid because of its relatively low viscosity, which produces a response time for the transducer of less than $6 \mathrm{~s}$. In order to enhance the magnitude of the pressure signal, experiments were generally run with 10-24 drops in a train. The additional pressure drop $\Delta P^{+}$for one drop was then obtained by dividing the total extra pressure drop by the total number of drops. It had previously been predicted by Hyman \& Skalak $(1972 a, b)$ for the Newtonian case that the pressure drop per drop should be independent of the spacing for separations of centres of at least one tube diameter. This was confirmed experimentally in the present study for both the Newtonian and viscoelastic cases.

Photographs giving the drop shape and streamlines were taken by cameras which moved parallel to the direction of motion of the drops. Those obtained for determination of the drop shape were taken using a $35 \mathrm{~mm}$ single-lens reflex camera with diffused background lighting and ASA 400 Tri-X film (Kodak). The streamline pictures were taken using a Graflex camera fitted for high-speed Polaroid Type 57 (3000 ASA) film. A $300 \mathrm{~W}$ projector lamp was allowed to shine through a slit $0.01 \mathrm{in}$. wide in a completely dark room. The slit was aligned lengthwise, parallel to the tube, so that the cross-sectional plane through the centre of the tube was illuminated for the pictures, which were taken in a horizontal direction. The motion of the fluid was traced by very small suspended particles, which appear as streaks in the pictures for exposure times of approximately $3 \mathrm{~s}$. Since the object (the suspended drop) was enclosed by a curved surface (the circular tube), we found that it was necessary to match the refractive indices of the suspending fluid and the fluid in the constant-temperature bath in order to minimize photographic distortions of the drop shape. The matching was considered adequate when the radii in different directions measured from a photograph of a spherical drop were found to differ by less than $1.5 \%$. Water was used in the bath when the suspending fluid was Separan in water. Aqueous sugar solution, $60 \%$ by weight, having a refractive index of 1.44 , was used in the bath when the suspending fluid was glycerine (refractive index 1.46).

\section{Experimental results}

In the present study we have measured $\Delta P^{+}, U$ and the drop shape for each of the eight different fluid systems listed in table 1 at four different flow rates and with six drop sizes in the range $0 \cdot 7 \lesssim \lambda \lesssim 1 \cdot 1$. The results are presented and discussed in the following four subsections. Although the discussion is primarily focused on the Newtonian case, comparison is also made, where appropriate, between the Newtonian and viscoelastic systems. In order to facilitate this comparison, the values of the drop viscosity were chosen to provide similar values of $\sigma$ for systems 1 and 5,2 and 6, 3 and 7, and 4 and 8. In addition, the values of $\Gamma$ for the viscoelastic system with volume flow rates $(b)$ and $(d)$ are very nearly the same as the values for flow rates $(a)$ and $(b)$, respectively, in the Newtonian system. The only experimental runs which are directly comparable with any of the available theoretical analyses are those for systems $2(b)$ and $(d)$, which have almost the same values of $\Gamma^{-1}(10$ and 4$)$ and $\sigma(1 \cdot 0)$ as were assumed in the analysis of Hyman \& Skalak (1972b). 


\subsection{Drop shape}

Photographs depicting the drop shape were taken for all the fluid systems, flow rates and drop volumes. For purposes of the present discussion, we reproduce those for systems $1(a), 1(b), 4(a), 4(b), 5(b), 5(d), 8(b)$ and $8(d)$ in figure 2 (plate 1). The remainder may be found in $H_{0}$ (1975). The shapes differ with variations in the flow rate $\bar{V}$, the viscosity ratio $\sigma$ and the drop size as measured by $\lambda$.

Let us first consider the variations in shape as $\lambda$ is changed, holding $\bar{V}$ and $\sigma$ constant. Obviously, in all cases, the length of the drop (i.e. the maximum dimension measured in the direction of the tube axis) increases with $\lambda$ since the volume of the drop is increased and the streamwise extent is unconstrained. As $\lambda$ increases, the maximum width (measured in the radial direction from the tube axis) first increases, but then, constrained by the wall, tends to become constant for $\lambda \gtrsim 1$. That is, the width of the layer of suspending fluid between the wall and the drop is essentially independent of the drop volume for $\lambda \gtrsim \mathbf{1} \cdot 0$. We shall see, in a later section, that the drop velocity also becomes independent of the drop volume as $\lambda$ is increased above $0 \cdot 9$. Both of these features were also observed by Goldsmith \& Mason (1963) in their investigation of the motion of very large bubbles.

Next we consider variations in shape caused by increasing the flow rate $\bar{V}$. It is evident from figure 2 that drops of the same volume and same $\sigma$, in both Newtonian and viscoelastic suspending fluids, become more elongated (in the direction of motion) as $\bar{V}$ increases. This increase in deformation with increased velocity is both intuitively obvious and in qualitative agreement with the theoretical predictions of Hetsroni et al. (1970) and Hyman \& Skalak (1972b).

Finally, we turn to the dependence of the drop shape on the viscosity ratio $\sigma$. For the Newtonian system, as $\sigma$ is increased with $\lambda$ and $\bar{V}$ held constant, the drops become more elongated, i.e. more viscous drops suffer a larger deformation. Although opposite to the intuitive notion that a more viscous drop should be less easily deformed, this result is in agreement with the perturbation theories of Hetsroni et al. (1970) [ef. equation (2)] and of Hyman \& Skalak (1972b). It should be noted however that both the theories and the present experiments are relevant only for viscosity ratios $\sigma$ of order unity, and one cannot, therefore, extrapolate to the obvious contradiction that a rigid sphere $(\sigma \rightarrow \infty)$ is more deformable than a gas bubble $(\sigma \rightarrow 0)$. Also, the shape change associated with a change in the viscosity ratio by a factor of ten is comparable in magnitude to that induced by a change in the velocity $\bar{V}$ of only $30 \%$. This more pronounced influence of $\Gamma$ on the shape, compared with $\sigma$, is again substantiated qualitatively by equation (2) (from Hetsroni et al. 1970). For the viscoelastic case, it is also clear from figure 2 that the more viscous drops are more elongated for given values of $\bar{V}$ and $\lambda$. However, unlike the Newtonian case, where the general drop shape is qualitatively similar for all values of $\sigma$, the drops in the viscoelastic fluid not only become more elongated with increasing $\sigma$, but also become increasingly pointed at the front and flattened at the back compared with drops for smaller $\sigma$, which have maximum girth somewhere near the middle (see figure 2).

It is also interesting to compare drops of the same $\sigma$ and $\Gamma$ for the Newtonian 
and viscoelastic systems. As shown in figure 2, for the case of high $\sigma$, on comparing system $1(a)\left(\sigma=2 \cdot 04, \Gamma^{-1}=13 \cdot 3\right)$ with system $5(b)\left(\sigma=3 \cdot 7, \Gamma^{-1}=13 \cdot 3\right)$ and system $1(b)\left(\sigma=2 \cdot 04, \Gamma^{-1}=9 \cdot 9\right)$ with system $5(d)\left(\sigma=4 \cdot 9, \Gamma^{-1}=10 \cdot 1\right)$, it may be seen that the drops in the viscoelastic systems appear to be more "streamlined' than those in the Newtonian systems. On the other hand, for the case of small $\sigma$, on comparing system $4(a)\left(\sigma=0 \cdot 19, \Gamma^{-1}=13 \cdot 3\right)$ with system $8(b)$ $\left(\sigma=0.13, \Gamma^{-1}=13 \cdot 3\right)$ and system $4(b)\left(\sigma=0 \cdot 19, \Gamma^{-1}=9.9\right)$ with system $8(d)$ $\left(\sigma=0 \cdot 17, \Gamma^{-1}=10 \cdot 1\right)$, it is apparent that the drops in the viscoelastic systems bulge and appear to be less 'streamlined' in shape compared with those in the Newtonian systems. It is also significant that for all $\sigma$ the layers of suspending fluid between the drops and wall are thicker in the viscoelastic systems than in the corresponding Newtonian cases.

\subsection{Streamlines}

In order to obtain a more detailed view of the dynamics of drop motion in tubes, a series of flow-visualization studies was made, based upon streamline pictures taken in the manner described in $\$ 2$. The camera was mounted on a platform which moved horizontally at precisely the same speed as the drops, which thus appear motionless in the pictures. Both Newtonian and viscoelastic suspending fluids were used. However, except for obvious variations resulting from the differences in drop shape, no qualitative differences could be detected between these two cases (see figure 3 , plate 2 ). In both, the fluid inside the drop recirculates with no net motion since the photographs were taken from a frame of reference which moved with the drop velocity. The motion of the suspending fluid has two distinct regimes. A central core of recirculating fluid is found between adjacent drops and centred about the tube axis with a radius approximately the same as the deformed drop radius. Since the entire central core recirculates, there is no net motion of this core of suspending fluid. That is, the average velocity inside this 'bolus' is the same as the drop velocity $U$. For this reason, the stagnation points in this core are located at about the same radial distance from the axis as the stagnation points inside the drop. Since $U / \bar{V}>1$, fluid in the second regime, which occupies a shell between the core and the tube wall, flows backwards relative to the drops. These qualitative features are similar to the observations of Goldsmith \& Mason (1963) and Prothero \& Burton (1961). The work of Prothero \& Burton (1961) was intended to show that the bolus flow helps to increase the mass transfer from the bulk fluid to the walls. Taylor (1960), who also reported a study of the motion of large bubbles in tubes, predicted the existence of a stagnation ring and stagnation vortex on the leading end of a bubble. This seems to be in agreement with our pictures since no motion of tracer particles is discernible in this region. Finally, a 'recirculating' core of suspending fluid is also found upstream and downstream of the leading and trailing drops of any train, including the case of a single drop.

\subsection{Drop velocity}

We now turn to the measured values of the drop velocity relative to the average overall fluid velocity of the system. Measurements were made for all combinations 


\begin{tabular}{|c|c|c|c|c|c|c|}
\hline$v(\mathrm{ml})$ & 0.2 & 0.3 & $0 \cdot 4$ & 0.5 & $0 \cdot 6$ & $0 \cdot 7$ \\
\hline \multicolumn{7}{|l|}{ System } \\
\hline $1(a)$ & 1.46 & $1 \cdot 35$ & $1 \cdot 27$ & 1.27 & 1.27 & 1.27 \\
\hline $1(b)$ & 1.46 & 1.35 & $1 \cdot 30$ & $1 \cdot 30$ & $1 \cdot 30$ & $1 \cdot 30$ \\
\hline $1(c)$ & 1.46 & $1 \cdot 35$ & $1 \cdot 32$ & $1 \cdot 32$ & $1 \cdot 32$ & 1.32 \\
\hline $1(d)$ & 1.47 & $1 \cdot 39$ & $1 \cdot 39$ & $1 \cdot 39$ & $1 \cdot 39$ & $1 \cdot 39$ \\
\hline $2(a)$ & 1.49 & $1 \cdot 36$ & 1.28 & $1 \cdot 28$ & $1 \cdot 28$ & 1.28 \\
\hline $2(b)$ & 1.51 & $1 \cdot 38$ & $1 \cdot 33$ & 1.33 & $1 \cdot 33$ & 1.33 \\
\hline $2(c)$ & 1.52 & 1.39 & $1 \cdot 36$ & $1 \cdot 36$ & $1 \cdot 36$ & $1 \cdot 36$ \\
\hline $2(d)$ & 1.53 & $1 \cdot 45$ & $1 \cdot 45$ & 1.45 & $1 \cdot 45$ & 1.45 \\
\hline $3(a)$ & 1.51 & $1 \cdot 37$ & $1 \cdot 29$ & 1.29 & 1.29 & 1.29 \\
\hline $3(b)$ & 1.52 & 1.41 & $1 \cdot 35$ & $1 \cdot 35$ & 1.35 & 1.35 \\
\hline $3(c)$ & 1.54 & $1 \cdot 42$ & $1 \cdot 39$ & $1 \cdot 39$ & 1.39 & 1.39 \\
\hline $3(d)$ & 1.58 & $1 \cdot 49$ & $1 \cdot 49$ & 1.49 & 1.49 & $1 \cdot 49$ \\
\hline $4(a)$ & 1.56 & 1.47 & $1 \cdot 39$ & $1 \cdot 39$ & 1.39 & 1.39 \\
\hline $4(b)$ & 1.57 & 1.48 & $1 \cdot 41$ & 1.41 & $1 \cdot 41$ & $1 \cdot 41$ \\
\hline $4(c)$ & $1 \cdot 59$ & $1 \cdot 50$ & 1.48 & 1.48 & 1.48 & 1.48 \\
\hline $4(d)$ & $1 \cdot 65$ & $1 \cdot 55$ & 1.55 & $1 \cdot 55$ & $1 \cdot 55$ & $1 \cdot 55$ \\
\hline $5(a)$ & $1 \cdot 37$ & $1 \cdot 31$ & $1 \cdot 26$ & $1 \cdot 26$ & $1 \cdot 26$ & $1 \cdot 26$ \\
\hline $5(b)$ & $1 \cdot 38$ & $1 \cdot 35$ & $1 \cdot 29$ & $1 \cdot 29$ & 1.29 & 1.29 \\
\hline $5(c)$ & 1.41 & $1 \cdot 35$ & $1 \cdot 32$ & $1 \cdot 32$ & $1 \cdot 32$ & 1.32 \\
\hline $5(d)$ & 1.47 & $1 \cdot 39$ & $1 \cdot 36$ & $1 \cdot 36$ & $1 \cdot 36$ & $1 \cdot 36$ \\
\hline $6(a)$ & $1 \cdot 37$ & $1 \cdot 31$ & $1 \cdot 26$ & $1 \cdot 26$ & $1 \cdot 26$ & 1.26 \\
\hline $6(b)$ & $1 \cdot 38$ & $1 \cdot 36$ & 1.29 & 1.29 & 1.29 & 1.29 \\
\hline $6(c)$ & 1.42 & 1.38 & $1 \cdot 32$ & 1.32 & 1.32 & $1 \cdot 32$ \\
\hline $6(d)$ & 1.47 & $1 \cdot 39$ & $1 \cdot 36$ & $1 \cdot 36$ & $1 \cdot 36$ & $1 \cdot 36$ \\
\hline$i(a)$ & $1 \cdot 38$ & $1 \cdot 31$ & $1 \cdot 28$ & $1 \cdot 28$ & $1 \cdot 28$ & 1.28 \\
\hline $7(b)$ & $1 \cdot 38$ & $1 \cdot 36$ & $1 \cdot 31$ & $1 \cdot 31$ & $1 \cdot 31$ & $1 \cdot 31$ \\
\hline $7(c)$ & 1.44 & $1 \cdot 38$ & $1 \cdot 36$ & $1 \cdot 36$ & $1 \cdot 36$ & $1 \cdot 36$ \\
\hline $7(d)$ & 1.49 & $\mathbf{1 . 3 9}$ & $1 \cdot 39$ & $1 \cdot 39$ & $1 \cdot 39$ & 1.39 \\
\hline $8(a)$ & $1 \cdot 40$ & $1 \cdot 33$ & $1 \cdot 32$ & $1 \cdot 32$ & $1 \cdot 32$ & $1 \cdot 32$ \\
\hline $8(b)$ & 1.42 & 1.38 & $1 \cdot 34$ & $1 \cdot 34$ & $1 \cdot 34$ & $1 \cdot 34$ \\
\hline $8(c)$ & $1 \cdot 47$ & 1.41 & 1.41 & 1.41 & 1.41 & 1.41 \\
\hline $8(d)$ & 1.51 & $1 \cdot 43$ & $1 \cdot 43$ & $1 \cdot 43$ & 1.43 & 1.43 \\
\hline \multicolumn{7}{|c|}{ TABLE 3. Relative velocity of drops $U / \vec{V}$} \\
\hline
\end{tabular}

of the viscosity ratio, drop size and volume flow rate in both the Newtonian and viscoelastic systems. The complete set of results is given in table 3 . As expected [cf. equation (1)], the drop velocity exceeds $\bar{V}$ in every case, generally being $30-60 \%$ faster. It is useful to consider the variations in $U / \bar{V}$ with respect to $\bar{V}$, $\lambda$ and $\sigma$ more closely.

We begin with the variation in $U$ with $\lambda$, holding $\bar{V}$ and $\sigma$ fixed. In both the viscoelastic and Newtonian systems, the increasing wall effect causes the drop velocity to decrease with increasing $\lambda$ until $\lambda \gtrsim 0 \cdot 9$, when the velocity becomes practically independent of $\lambda$. As we have noted previously, the latter observation is in agreement with the results of Goldsmith \& Mason (1963), and is presumably a result of the independence of the drop cross-section of $\lambda$ which was noted in $\S 3.1$. In order to compare the present experimental results with the available 


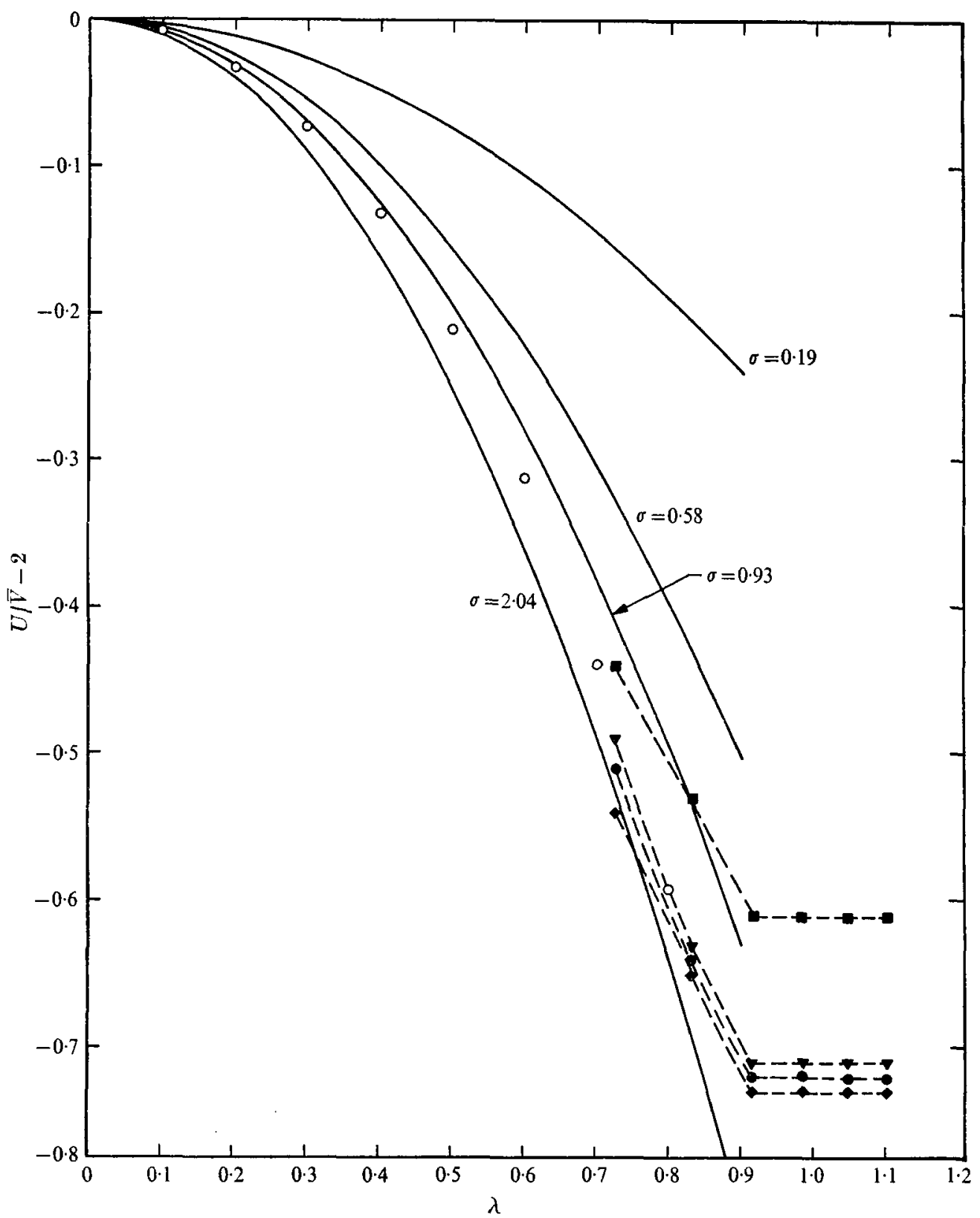

Figure 4(a). For legend see next page.

theory, we have plotted the quantity $U / \bar{V}-2$ in figure $4(a)$ as a function of $\lambda$ for the four Newtonian systems at the lowest flow rate $(a)$, where deformation is least important. Also shown are the theoretical predictions from (1) (from Hetsroni et al. 1970) at the same viscosity ratios, and the numerical results of Hyman \& Skalak (1972 $a$ ) for $\sigma=1$ and no shape deformation. Equation (1), 


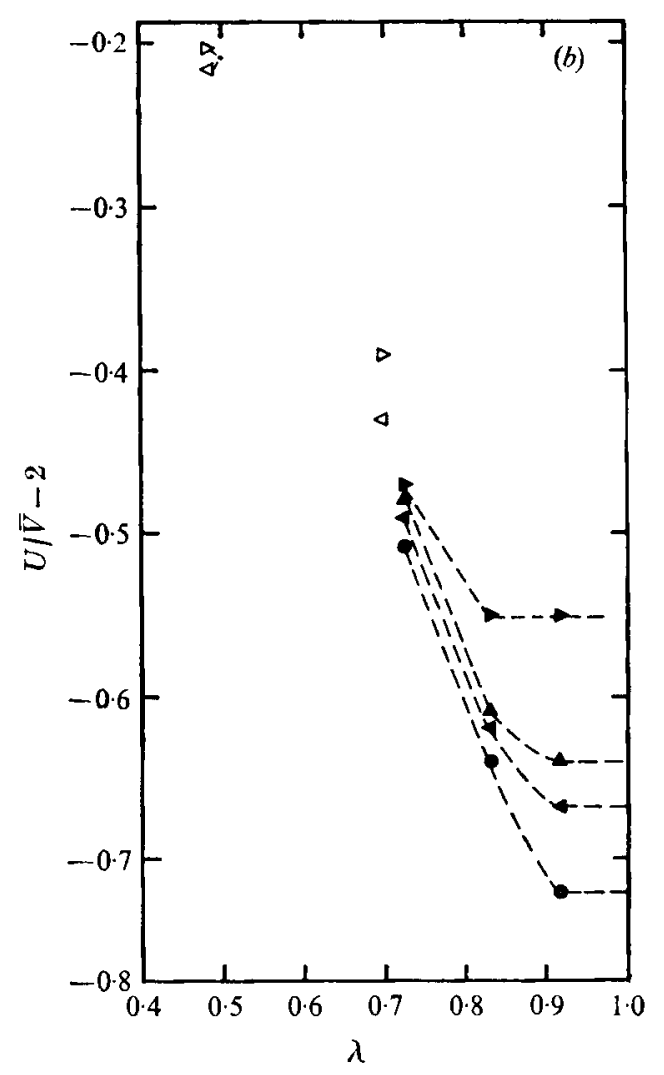

Figure 4. Relative velocity of drops $U / \bar{V}-2$ vs. drop size. $(a)$, system $1(a) ; 0$, system $2(a) ; \boldsymbol{\nabla}$, system $3(a)$; , system $4(a)$; $\bigcirc$, results of Hyman \& Skalak $(1972 a)$ for undeformed drops and $\sigma=1$; — equation (1) (Hetsroni et al. 1970) for $\sigma=2.04,0.93$, 0.58 and $0 \cdot 19 .(b) \boldsymbol{O}$, system $2(a) ; \boldsymbol{\Lambda}$, system $2(b) ; \boldsymbol{\Delta}$, system $2(c) ; \boldsymbol{D}$, system $2(d)$; $\triangleleft, \triangleright$, results of Hyman \& Skalak (1972b) for deformed drops, $\sigma=1$ and $\Gamma^{-1}=10$ and 4 respectively.

which is strictly valid only for $\lambda \ll 1$, predicts too high values for $C^{r} / \bar{V}$ at the moderate values of $\lambda$ which characterize the experiments. On the other hand, the agreement with the results of Hyman \& Skalak (1972a) is quite good. Comparing results for the Newtonian and viscoelastic systems, it may be noted that the rate of decrease of $U / \bar{V}$ with increasing $\lambda$ is more rapid for the Newtonian case. As a consequence, for comparable values of $\sigma$ and $\Gamma$, drops move faster in the Newtonian system than in the viscoelastic system for $\lambda \lesssim 0 \cdot 8$, but slower for $\lambda \gtrsim 0 \cdot 9$.

The relative drop velocity $U / \bar{V}$ increases with increasing average fluid velocity for both the Newtonian and viscoelastic cases. That is, more rapid relative drop motion is associated with larger deformation. This trend is in qualitative agreement with the available theoretical results of Hyman \& Skalak (1972b) as may be seen from figure $4(b)$, where we have plotted $U / \bar{V}-2 v s$. $\lambda$ for the Newtonian system $2(\sigma=0.93)$ for all four flow rates $(a)-(d)$, together with Hyman \& skalak's $(1972 b)$ calculated values for $\sigma=1, \Gamma^{-1}=4$ and 10 , and $\lambda=0 \cdot \tilde{5}$ and $0 \cdot 7$. 


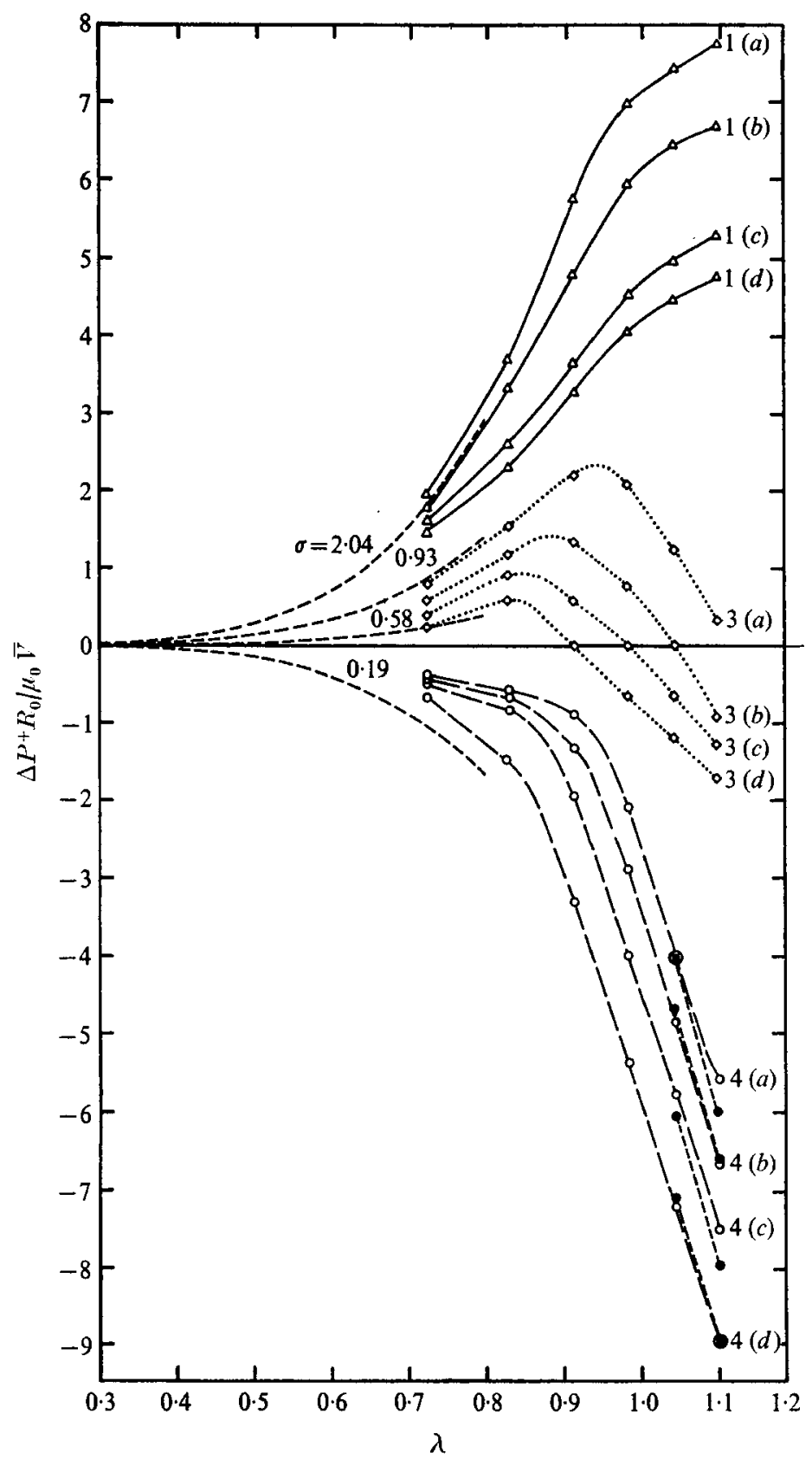

Figure 5. Dimensionless extra pressure drop $\Delta P+R_{0} / \mu_{0} \bar{V} v s . \lambda$ for systems $1(a)-(d)$, $3(a)-(d)$ and $4(a)-(d)$; ———, equation (3) (Brenner 1971) for $\sigma=2.04,0.58$ and $0 \cdot 19$; ..., estimation of extra pressure drop by assuming that an increase in $\lambda$ is equivalent to an increase in an annular core starting from $\lambda=0.985$. 


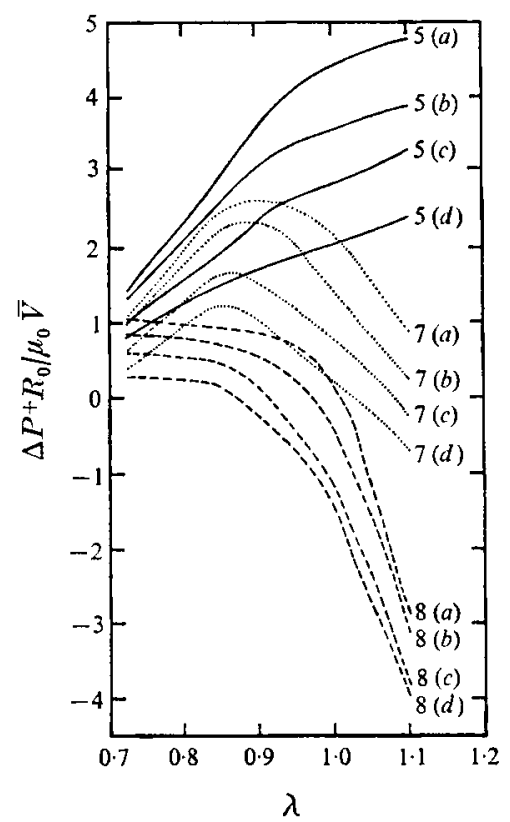

Figure 6. Dimensionless extra pressure drop $\Delta P+R_{0} / \mu_{0} \bar{V}$ vs. $\lambda$ for systems $5(a)-(d), 7(a)-(d)$ and $8(a)-(d)$.

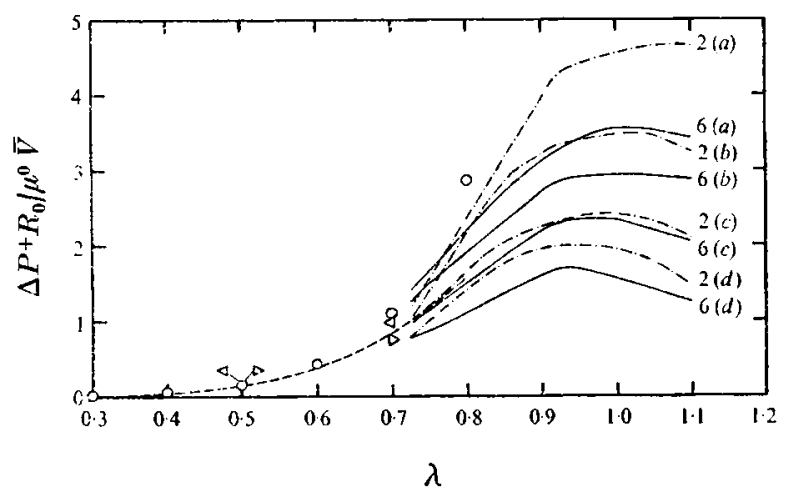

FIgURE 7. $\Delta P^{+} R_{0} / \mu_{0} \bar{V} v s$. $\lambda$ for systems $2(a)-(d)$ and $6(a)-(d)$. - - - equation (3) for $\sigma=0.93$. Results of Hyman \& Skalak $(1972 a, b)$ for $\sigma=1: 0$, undeformed drops; $\triangleleft, \Gamma^{-1}=10$, deformed drops; $\triangleright, \Gamma^{-1}=4$, deformed drops.

Finally, we turn to the variation in $U / \bar{V}$ with $\sigma$, holding $\lambda$ and $\bar{V}$ constant. The experimental results in both the Newtonian and viscoelastic systems show that $U / \bar{V}$ increases as $\sigma$ decreases. Unlike the particle deformation, however, for which variations in $\sigma$ are relatively unimportant compared with variations in $\bar{V}$, the effect of $\sigma$ on $U / \bar{V}$ is comparable to that of $\bar{V}$. As is obvious from figure $4(a)$, theory and experiment are in qualitative agreement with regard to the dependence of $U / \vec{V}$ on $\sigma$. 


\subsection{Extra pressure drop $\Delta P^{+}$}

The quantity of most interest from the technological point of view is the change $\Delta P^{+}$in the pressure drop owing to the presence of suspended drops, relative to that which would occur at the same volume flow rate with the suspending fluid alone. The various experimental results are summarized in figure 5 (Newtonian systems 1,3 and 4), figure 6 (viscoelastic systems 5,7 and 8) and figure 7 (systems 2 and 6), where we have plotted the dimensionless quantity $\Delta P^{+} R_{0} / \mu_{0} \bar{V}$ as a function of $\lambda$ for various combinations of $\sigma$ and the flow rate. Shown along with the Newtonian results are calculated values of $\Delta P+R_{0} / \mu_{0} \bar{V}$ taken from the small- $\lambda$ theory for undeformed drops of Brenner (1971) [see (3)]. Before discussing these results in detail, it is useful to summarize the various physical phenomena which can affect the magnitude and sign of the extra pressure drop.

These separate logically into three distinct mechanisms: the simple exchange of suspending fluid with drop fluid of different viscosity, which in principle would be active even if the flow field were unchanged; the alteration of the flow field due to the presence of the drop interface; and the alteration of the drop shape, leading to changes in the flow. Brenner's (1971) analysis of an undeformed drop demonstrates the nature of the interaction of the first two mechanisms for a moderately small drop $(\lambda \ll 1)$. In particular, even when $\sigma=1$, so that the first mechanism is not active, Brenner's solution shows that the extra dissipation associated with modification of the flow field still gives $\Delta P^{+}>0$. Only when $\sigma$ is decreased to approximately 0.48 does the replacement of more viscous with less viscous fluid overtake the effect of altered flow to give $\Delta P^{+}=0$. The effect of drop deformation for these moderate values of $\lambda$ is to decrease $\Delta P+$ from its value for the undeformed state. Thus any of the features producing larger deformation (see $\$ 3.1$ ) will produce also a tendency towards lower $\Delta P^{+}$. It should be noted that, in many instances, the various effects will be competing, so that $\Delta P^{+}$may either increase or decrease depending on the relative importance of each. For example, an increase in $\bar{V}$ with $\sigma$ and $\lambda$ constant was previously seen to give increased deformation and thus by the present argument gives a decrease in $\Delta P^{+}$. However, an increase in $\sigma$ with $\bar{V}$ and $\lambda$ fixed also increases the deformation, but at the same time increases the average $\mu$ in the system and causes some alteration in the flow fields in and around the drop. In view of the weak dependence of the shape on $\sigma$, it should be expected that the latter effects will dominate, thus producing increased values of $\Delta P^{+}$. Finally, it should be noted that, as $\lambda$ increases, the increased wall effect will tend to cause $\Delta P+$ to increase as $\lambda^{5}$ for small $\lambda$ [see (3)]. However, as $\lambda$ is increased near unity, the drop shape is constrained by the presence of the walls, and in fact we have seen that the main effect of increasing volume is to increase the length of the drop with other geometric features remaining reasonably unchanged. Indeed, for $\lambda>1$, it might be expected that the differential change in $\Delta \boldsymbol{P}^{+}$with $\lambda$ is approximately that associated with an increase in length of an annular flow region for two fluids of different viscosity. This suggests, therefore, that for large $\lambda$ the main effect on $\Delta P^{+}$is equivalent to simple replacement of one fluid with another of different viscosity. Furthermore, it can be anticipated that in this simplified regime $\Delta P^{+}$will increase with increasing 


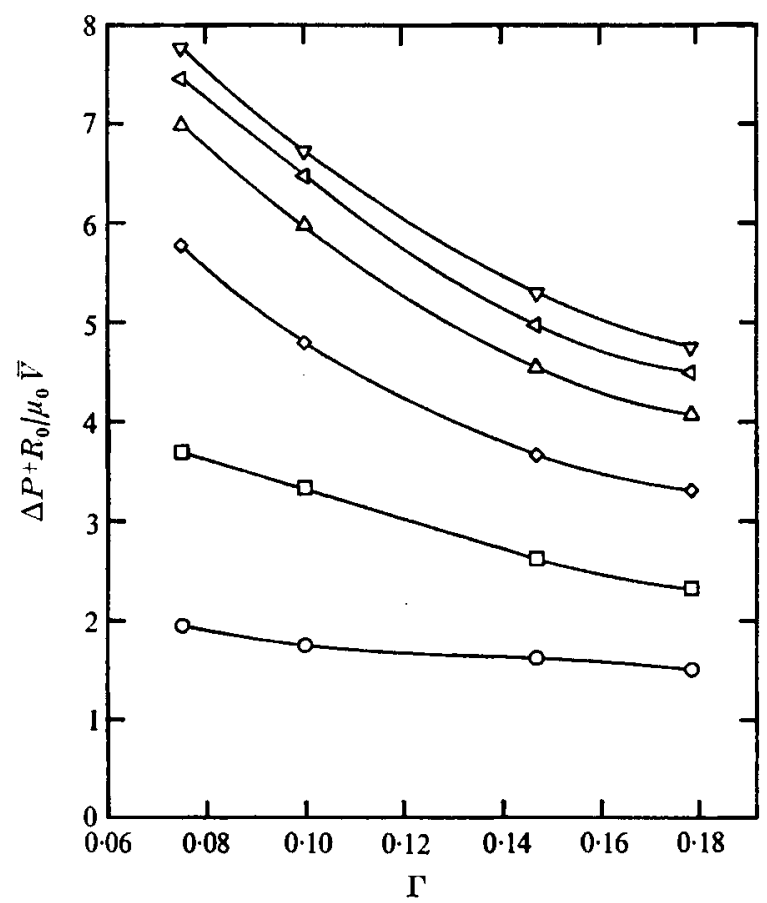

FigURE 8. $\Delta P+R_{0} / \mu_{0} \vec{V}$ vs. $\Gamma\left(=\mu_{0} \bar{V} / \gamma\right)$ for system 1. $O, \lambda=0.726 ; \square, \lambda=0.831$; $\diamond, \lambda=0.914 ; \Delta, \lambda=0.985 ; \triangleleft, \lambda=1.046 ; \nabla, \lambda=1.102$.

$\lambda$ for $\sigma>1$ and decrease with increasing $\lambda$ for $\sigma<1$. Thus for intermediate $\lambda$ we may anticipate a transition in the value of $\sigma$ corresponding to $\Delta P^{+}=0$ from $\sim 0.48$ to 1 .

Let us now consider the detailed experimental results in the light of these qualitative physical ideas.

Newtonian fluid systems. The most straightforward variations are those for different values of $\sigma$, holding the flow rate and drop size $\lambda$ fixed. The general trend is consistent with theoretical expectations: for the two largest values of $\sigma$, an added pressure drop is always positive, while for the smallest value $(\sigma=0 \cdot 19)$ it is always negative. The intermediate value $(\sigma=0.58)$ corresponds to both positive and negative values depending on the flow rate and on $\lambda$. These results may be compared qualitatively with the theoretical prediction of Brenner (1971) that $\Delta P^{+}=0$ for $\sigma \simeq 0.48$, assuming small $\lambda$ and no deformation. Although the experimental results are complicated by the effects of particle deformability and varying degrees of wall effect for the larger values of $\lambda$, it is nevertheless elear from this comparison that the main effect of varying $\sigma$ is, as anticipated in the introduction to this section, simply the replacement of fluid with drop fluid of different viscosity, which of course also affects the flow field. The third mechanism, resulting from increased deformation with increasing $\sigma$, would actually tend to cause a decrease in $\Delta P+$ for a more viscous drop. However, it is obvious from the experimental results that this change in deformation is so small that its effect on $\Delta P+$ is very much dominated by the first two mechanisms. 
Like the dependence on $\sigma$, the dependence of $\Delta P^{+}$on the flow rate is relatively straightforward. Indeed, for fixed $\sigma$ and $\lambda, \Delta P^{+} R_{0} / \mu_{0} \bar{V}$ decreases with increasing flow rate in every case. That is, the behaviour of the pressure drop is similar to that in a shear-thinning fluid. This is perhaps more clearly illustrated in figure 8 , where we have plotted $\Delta P+R_{0} / \mu_{0} \bar{V}$ as a function of the flow-rate parameter $\Gamma \equiv \mu_{0} \bar{V} / \gamma$ for $\sigma=2 \cdot 04$ and various values of $\lambda$. As we have suggested in the introduction to this section, the dependence of $\Delta P^{+}$on the flow rate (or $\Gamma$ ) is primarily a reflexion of the dependence of the drop shape on $\Gamma$. It is evident from figure 8 , which is typical of all four Newtonian fluid systems, that the dependence of the additional pressure drop on $\bar{V}$ (or $\Gamma$ ) is not very significant for small drop volumes (low values of $\lambda$ ), but that the variation in $\Delta P^{+}$with the flow rate becomes very prominent for larger values of $\lambda$ (larger drops). This behaviour is consistent with the observations which we have reported of the drop shape. Thus for small volumes, especially $v=0.2 \mathrm{ml}(\lambda=0.726)$, the shape is not significantly altered as the flow rate is changed, while for larger volumes, the change in shape with flow rate is much more prominent.

Compared with the influences of $\sigma$ and $\Gamma$ on $\Delta P^{+}$, its dependence on the drop size $\lambda$ is more complicated. This is because changes in the drop size are associated with several competing mechanisms for variation in $\Delta P^{+}$. Assuming that the drops are either spherical or only slightly deformed, the two main effects of increasing $\lambda$ are due to the increased volume of the suspended drop and the increased wall effect (effectively, the decrease in the cross-sectional area of the channel which is available for the suspending fluid). This is indeed the case for values of $\lambda \simeq 0 \cdot 7$, for which our results are seen to behave similarly to the predictions of Brenner (1971), who included only these two mechanisms. The experimental results for system 2 are plotted in figure 6 , together with the corresponding theoretical results of Brenner (1971) and the results of Hyman \& Skalak $(1972 a, b)$ for an undeformed drop with $\sigma=1.0$ and $\lambda \geqslant 0.8$ and deformable drops with $\sigma=1 \cdot 0, \Gamma^{-1}=4$ and 10 and $\lambda=0.5$ and $0 \cdot 7$. Given the slight differences in $\sigma$ and $\Gamma$ between the experiments and the Hyman-Skalak theory, the comparison is quite satisfactory.

As $\lambda$ increases towards order unity, the drops are increasingly deformed from the spherical shape, and for cases with $\Delta P^{+}>0$, the rate of increase of $\Delta P^{+}$with $\lambda$ is decreased. Similarly, for the case $\Delta P^{+}<0, \Delta P^{+}$decreases more rapidly. This is the 'transition region' in our experiments, where the rate of change of $\Delta P^{+}$with $\lambda$ begins to deviate from the behaviour predicted by (3).

The behaviour for $\lambda>1$ requires more careful consideration. Although the drops are more deformed from a spherical shape for larger values of $\lambda$, the main change, as we have noted earlier, is an increase in length. Specifically, the changes in geometry at the front and back of the drop are relatively small and it is mainly the region of 'constant' cross-sectional area which is increased. Thus the change in the detailed flow structure with increasing $\lambda$ for $\lambda \gtrsim 1$ is almost totally confined to an increase in the length of the 'annular' flow region at the middle of the drop. For a true annular flow of two immiscible fluids, the pressure drop is increased with increasing $\lambda$ for $\sigma>1$ and decreased for $\sigma<1$. We suggest that the measured increments of $\Delta P^{+}$with increasing $\lambda$ for $\lambda \geqslant 1$ can be interpreted essentially as 
being due to an increase in length of an (admittedly complicated) 'annular' region. This would imply, as suggested in the introduction of this section, that $\Delta P^{+}$should increase with increasing $\lambda$ for $\lambda \gtrsim 1$ and $\sigma>1$, but decrease for $\sigma<1$. This qualitative idea is in complete agreement with the experimental results for $\lambda \gtrsim 1$. Thus for system $2(\sigma<1)$ the extra pressure drop increases with $\lambda$ for $\lambda \lesssim 1$, but does decrease for $\lambda \gtrsim 1$ as expected. Similarly for system $3(\sigma=0 \cdot 58)$ the extra pressure drop, which is initially positive and increasing for $\lambda \lesssim 1$, reaches a maximum at $\lambda=1$, and then decreases to negative values for $\lambda \gtrsim 1$. The same argument can be applied to systems 1 and 4 to explain the behaviour of $\Delta P+$ for $\lambda \gtrsim 1$. In particular, we have found that $\Delta P^{+}$increases at a decreasing rate for system 1 but at an increasing rate for system 4 in the region $\lambda \gtrsim 1$. Surprisingly, it appears that we can actually estimate the increase (or decrease) in the extra pressure drop for a change in $\lambda$ by assuming that the increase in $\Delta P^{+}$ is due solely to an increase in the length of an annular core of the suspended fluid. This increase (for $\sigma>1$ ) or decrease $(\sigma<1)$ in the additional pressure drop can be shown to be

$$
-8 \Delta L\left(\frac{\beta^{4}}{\beta^{4}+\sigma /(1-\sigma)}\right),
$$

where $\Delta L$ is the increase in the length of the drop and $\beta$ is the radial distance of the interface from the tube axis. Both quantities are non-dimensionalized by the tube radius $R_{0}$. An example is given for system 4 (see figure 5), where this effect on $\Delta P^{+}$is assumed to hold starting from $\lambda=0.985$ and values of $\Delta L$ and $\beta$ are obtained from the drop-shape photographs.

Viscoelastic fluid systems. Let us now consider the contribution of viscoelasticity to the extra pressure drop. Qualitatively, a comparison of the results for systems 5-8 in figures 5 and 7 with those for systems 1-4 in figures 6 and 7 shows surprisingly little difference when $\Delta P^{+}$is non-dimensionalized with respect to the characteristic wall-shear viscosity for simple tube flow of the viscoelastic fluid at the same flow rate. Indeed, nearly all of the detailed discussion above for systems 1-4 may be carried over to the viscoelastic case. 'The only major exception is the magnitude of $\Delta P+R_{0} / \mu_{0} \bar{V}$, which appears to be somewhat lower than for the Newtonian problem at larger $\sigma$, but somewhat larger (less negative) in the case of low $\sigma$.

This last point is illustrated in figure 9 , where we have compared results for equal values of the deformation (flow-rate) parameter $\Gamma^{-1}(\mathbf{1 3 \cdot 3})$ and approximately equal pairs of $\sigma$ values for corresponding Newtonian and viscoelastic case. The extra pressure drop is considerably lower for $\sigma=3.7$ in the viscoelastic fluid than for $\sigma=2.04$ in the Newtonian fluid. Similar behaviour is observed for $\sigma \simeq 1$, although the difference is less pronounced. Finally, for $\sigma \simeq 0 \cdot 15$, the situation is reversed, i.e. the viscoelastic fluid actually has a larger value of $\Delta P+R_{0} / \mu_{0} \bar{V}$ than the Newtonian fluid. At present, we can give no firm explanation for this phenomenon. We simply recall that a substantial transition in the qualitative shape was also observed for the viscoelastic fluid (compared with the Newtonian case) as $\sigma$ was varied, and it seems likely that the two features are connected. However, any simple-minded argument based on the shape of the drops without 


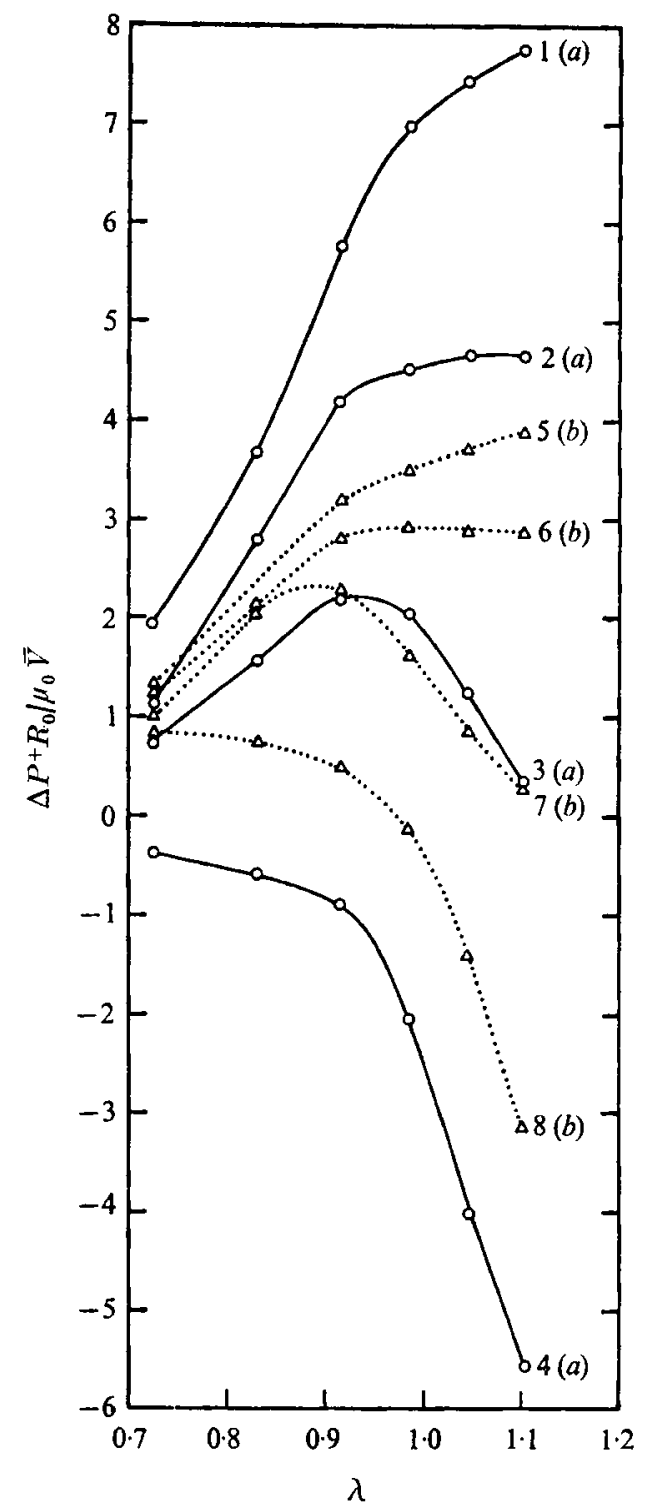

Figure 9. A comparison of extra pressure drops for Newtonian and viscoelastic systems for $\Gamma^{-1}=13 \cdot 3$.

consideration of the actual flow field and of other properties of the viscoelastic fluid (e.g. shear thinning, normal stresses or extensional viscosity) would be fortuitous, and of little fundamental value.

\section{Conclusion}

We have presented results for the additional pressure drop, the drop velocity, the drop shape and the streamlines for a train of neutrally buoyant drops suspended concentrically in a cylindrical tube. Reasonable agreement was found on 
comparing available theoretical and experimental reports in the literature with our present work on the limiting behaviour of the drop shape, $U / \bar{V}$ and $\Delta P^{+}$at high and low values of $\lambda$ and at certain intermediate values. Also, we were able to explain qualitatively the results for intermediate values of $\lambda$. This study also points out the differences observed between the cases of a viscoelastic and a Newtonian suspending fluid. In particular, the use of a viscoelastic fluid caused a substantial transition in the shape of the drops (compared with the Newtonian case) as the viscosity of the suspended drop was varied, and caused $\Delta P^{+} R_{0} / \mu_{0} \bar{V}$ to be reduced for high values of $\sigma(\gtrsim 0.58)$ but increased for low values of $\sigma$ compared with the Newtonian case. It may be noted, however, that in comparing the viscoelastic and Newtonian systems the former was characterized only by an apparent viscosity at the wall shear rate which is relevant for simple tube flow of the pure suspending fluid. A systematic variation of the viscoelastic properties (and also purely viscous properties, e.g. shear thinning) was not attempted and probably such an attempt is necessary before any qualitative explanation on the role of viscoelasticity can be achieved. Nevertheless the present study should be helpful in understanding two-phase flow in porous media, transport of twophase fluids in tubes and the macroscopic modelling of blood flow in capillaries, and moreover, induce further study in this area of research.

This work was supported, in part, by NSF Grant ENG 74-17590, and, in part, by Grant 6489-AC7 from the Petroleum Research Fund, administered by the American Chemical Society.

\section{REFERENCES}

Bresner, H. 1971 Pressure drop due to the motion of neutrally buoyant particles in duct flows. II. Spherical droplets and bubbles. Indust. Engng Chem. Fund. 10, 537.

Bretherton, F. P. 1961 The motion of long bubbles in tubes. J. Fluid Mech. 10, 166. Coleman, B. D., Markovitz, H. \& Noll, W. 1966 Viscometric Flows on Non-Neutomian Fluids. Springer.

Goldsurit, H. L. \& Mason, S. G. 1963 The flow of suspensions through tubes. II. Single large bubbles. J. Colloid Sci. 18, 237.

Hetsroni, G., Haber, S. \& Wacholder, E. 1970 The flow field in and around a droplet moving axially within a tube. J. Fluid Mech. 41, 689 .

Ho, B. P. 1975 Ph.D. thesis, California Institute of Technology.

Hochмuth, R. M. \& Sutera, S. P. 1970 Spherical caps in low Reynolds-number tube flow. Chem. Engng Sci. 25, 593.

Huppler, J. D., Ashare, E. \& Holmes, L. A. 1967 Rheological properties of three solutions. Part I. Non-Newtonian viscosity, normal stresses, and complex viscosity. Trans. Soc. Rheol. 11, 159.

Huppler, J. D., MacDonald, I. F., Asifare, E., Spriggs, T. W., Bird, R. B. \& Holmes, L. W. 1967 Rheological properties of three solutions. Part II. Relexation and growth of shear and normal stresses. Trans. Soc. Rheol. 11, 181.

Hyman, W. A. \& Stratak, R. $1972 a$ Viscous flow of a suspension of liquid drops in a cylindrical tube. Appl. Sci. Res. 26, 27.

Hyman, W. A. \& Skatak, R. $1972 b$ Non-Newtonian behavior of a suspension of liquid drops in tube flow. A.I.Ch.E. J. 18, 149.

Karnis, A. \& Mason, S. G. 1967 Particle motions in sheared suspensions. XXIII. Wall migration of fluid drops. J. Colloid Interface Sci. 24, 164. 
Leat, L. G., Skoog, J. \& Acrivos, A. 1971 On the motion of gas bubbles in a viscoelastic fluid. Can. J. Chem. Engng, 49, 569.

Prothero, J. \& Burton, A. C. 1961 The physics of blood flow in capillaries. I. The nature of the motion. Biophys. J. 2, 199.

Prothero, J. \& Burton, A. C. 1962 The physics of blood flow in capillaries. III. The pressure required to deform erythrocytes in acid-citrate-dextrose. Biophys. J. 2, 213.

Savins, J. G. 1969 Non-Newtonian flow through porous media. Indust. Engng Chem. 61, 18.

Sutera, S. P. \& Hochmuth, R. M. 1968 Large scale modeling of blood flow in the capillaries. Biorheol. 5, 45.

TAYLOR, G. I. 1960 Deposition of a viscous fluid on the wall of a tube. J. Fluid Mech. $10,161$. 


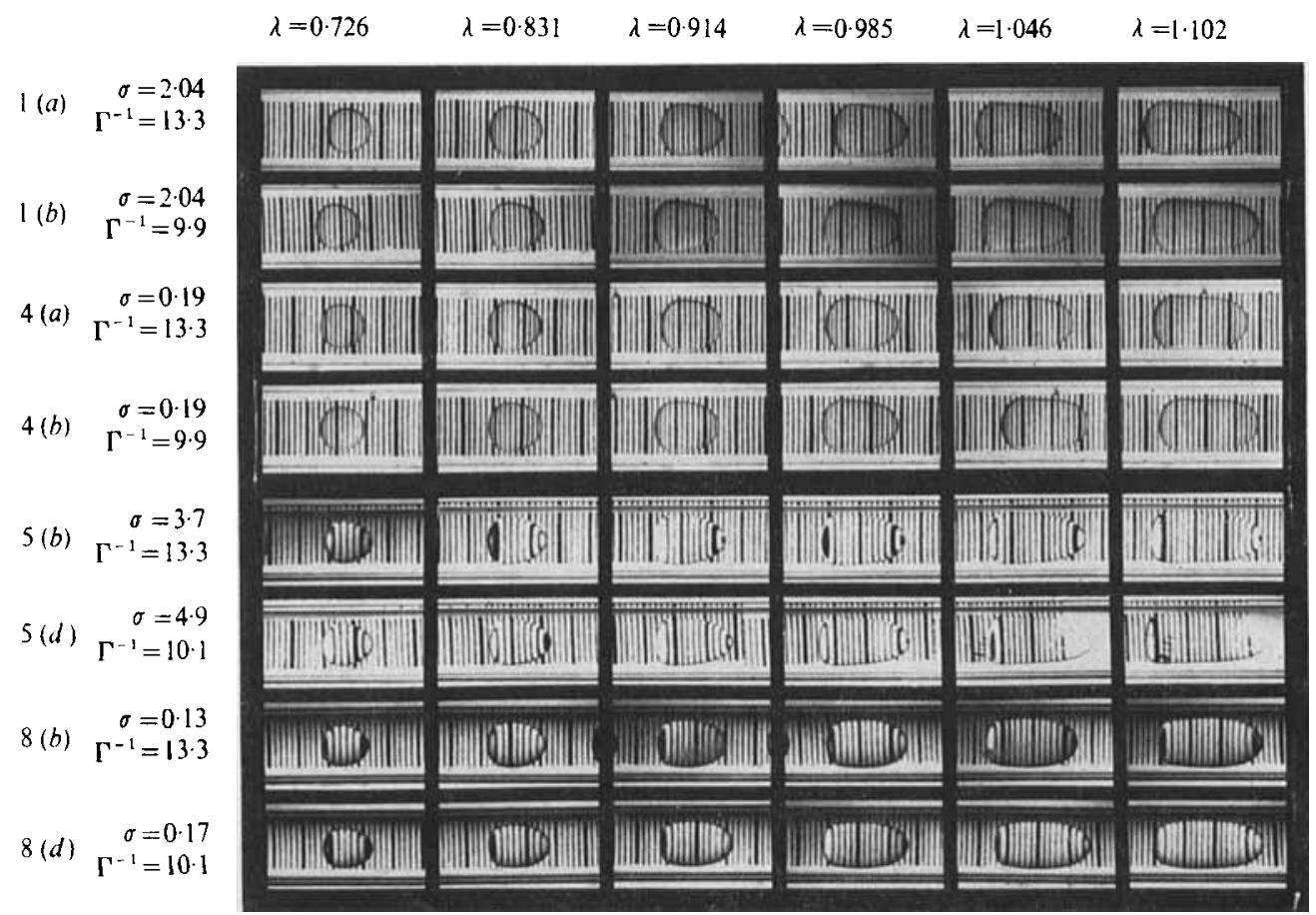

F'IGURe 2. Drop shapes for systems $1(a), 1(b), 4(a), 4(b), 5(b), 5(d), 8(b)$ and $8(d)$. 


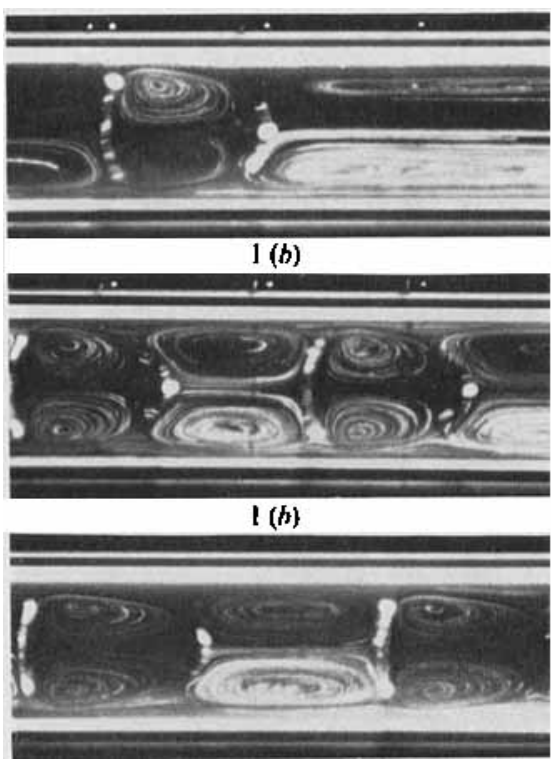

$2(d)$

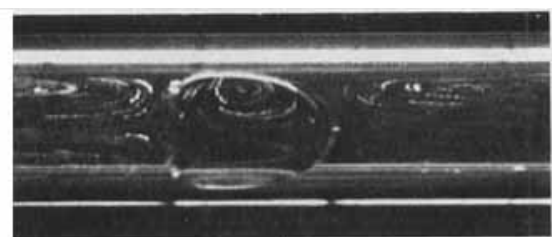

$7(b)$

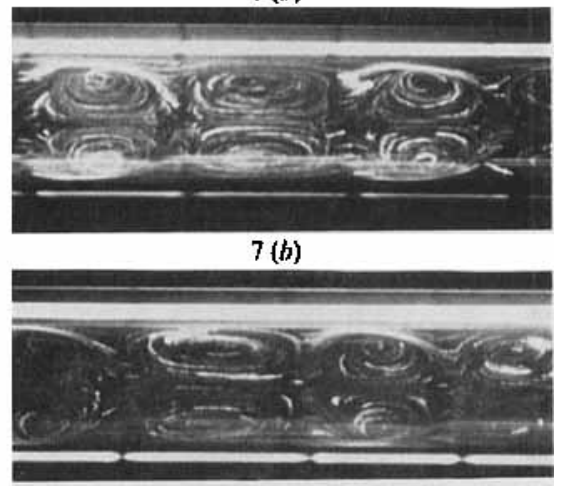

$6(h)$

Figure 3. Streamlines for system $1(b)$, a single drop and the leading drop of a train: system $2(d)$, a train of drops; system $6(b)$, a train of drops; and system $7(b)$, a single drop) and a train of drops. 